\title{
Textbook of Pediatric Rheumatology, 6th Edition
}

James T. Cassidy, Ross E. Petty, Ronald Laxer, Carol Lindsley. Philadelphia: Saunders/Elsevier, October 2010, 794 pages, $\$ 199$ US

This is an encyclopedic textbook covering the major areas of interest to pediatric rheumatologists. As such it suffers from the problems of any encyclopedic work. While virtually every area is discussed, there are more complete and more up to date discussions in more focused texts and more recent review articles. This is particularly true of the basic science sections. With 50 chapters and 64 authors, the quality of the information and the writing varies widely. While many diseases such as psoriatic arthritis, systemic lupus erythematosus, and sarcoidosis may occur in children, they are not in fact pediatric diseases. The chapters on these subjects in adult rheumatology texts are generally more complete and informative, although this text provides a more comprehensive collection of references relevant to pediatrics. Unfortunately, many references are cited but the actual reference has been omitted from the book, only to be found in the online edition. Some chapters are excellent, such as the section on juvenile dermatomyositis, which is truly different in children.

In dealing with childhood arthritis, the text is inconsistent in referring to "juvenile idiopathic arthritis" in some chapters and "juvenile rheumatoid arthritis" in others. Throughout, there is a failure to acknowledge that this is not a single disease entity, rather a collection of disparate diseases, making the discussions of a single etiology or pathogenesis unrevealing. What makes pediatric rheumatology special are the skills required to deal with children and their families. This should be a special emphasis of this book, but the information provided falls far short of the need. There is only a single paragraph on adherence, and much of the chapter on managing children is devoted to theoretical modeling without practical application. Indeed only 8 pages of nearly 800 in this text are devoted to what truly makes being a pediatric rheumatologist a unique skill. Pediatric rheumatologists will need this book on their shelves for reference and for examination preparation. Like an encyclopedia it provides a comprehensive overview, but it does not provide the knowledge or promote the skills required to successfully practice pediatric rheumatology.

THOMAS LEHMAN, MD, Pediatric Rheumatology, Hospital for Special Surgery, 535 East 70th Street, New York, New York 10021, USA.

E-mail: goldscout@aol.com

J Rheumatol 2011;38:10; doi:10.3899/jrheum.110630 